\title{
Exploring the Well-being of Health Care Workers During the COVID-19 Pandemic: Protocol for a Prospective Longitudinal Study
}

Jenny J W Liu ${ }^{1,2}$, PhD; Anthony Nazarov ${ }^{1,2}$, PhD; Rachel A Plouffe ${ }^{1,2}, \mathrm{PhD}$; Callista A Forchuk ${ }^{1}$, MSc; Erisa Deda ${ }^{1}$, MHIS; Dominic Gargala ${ }^{1}$, BSc; Tri Le ${ }^{1}$, BHSc; Jesse Bourret-Gheysen ${ }^{1}$, BAT; Vanessa Soares ${ }^{1}$, MD; Maede S Nouri ${ }^{1}$, MSc; Fardous Hosseiny ${ }^{3}$, MSc; Patrick Smith ${ }^{3}$, PhD; Maya Roth ${ }^{4}$, PhD, CPsych; Arlene G MacDougall ${ }^{2}$, MD; Michelle Marlborough $^{2,4}$, MD; Rakesh Jetly ${ }^{5}$, MD, OMM, CD; Alexandra Heber ${ }^{6}$, MD; Joy Albuquerque ${ }^{7}$, MD; Ruth Lanius ${ }^{2}$, MD, PhD; Ken Balderson ${ }^{2,4}$, MD, CM; Gabrielle Dupuis ${ }^{3}$, MSc; Viraj Mehta ${ }^{4}$, MD; J Don Richardson ${ }^{1,2,4}$, MD

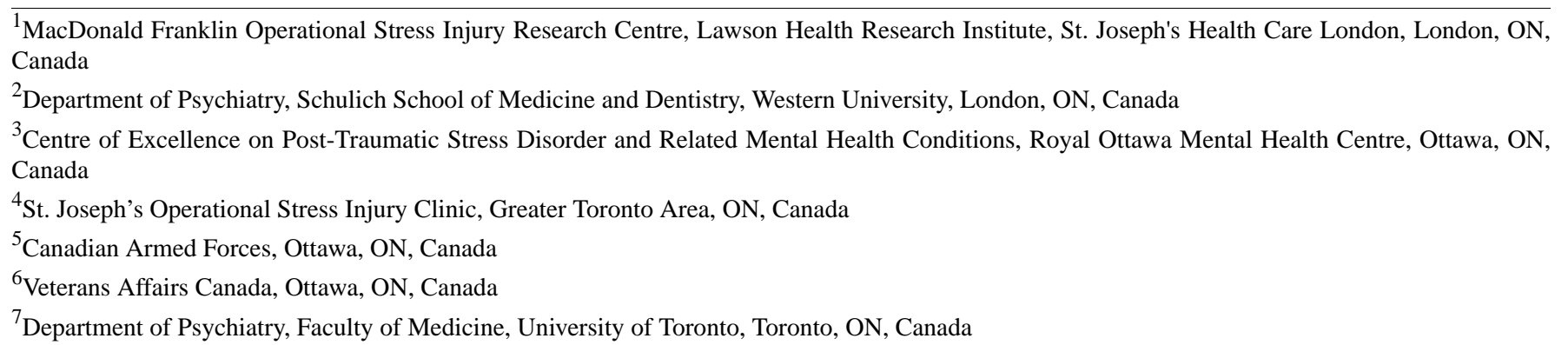

\section{Corresponding Author:}

Jenny J W Liu, PhD

MacDonald Franklin Operational Stress Injury Research Centre

Lawson Health Research Institute

St. Joseph's Health Care London

Parkwood Institute Research, Mental Health Building RM F4-367

550 Wellington Road

London, ON, N6C 0A7

Canada

Phone: 15196854292 ext 48211

Email: jenny.liu@sjhc.london.on.ca

\section{Abstract}

Background: Health care workers (HCWs) have experienced several stressors associated with the COVID-19 pandemic. Structural stressors, including extended work hours, redeployment, and changes in organizational mandates, often intersect with interpersonal and personal stressors, such as caring for those with COVID-19 infections; worrying about infection of self, family, and loved ones; working despite shortages of personal protective equipment; and encountering various difficult moral-ethical dilemmas.

Objective: The paper describes the protocol for a longitudinal study seeking to capture the unique experiences, challenges, and changes faced by HCWs during the COVID-19 pandemic. The study seeks to explore the impact of COVID-19 on the mental well-being of HCWs with a particular focus on moral distress, perceptions of and satisfaction with delivery of care, and how changes in work structure are tolerated among HCWs providing clinical services.

Methods: A prospective longitudinal design is employed to assess HCWs' experiences across domains of mental health (depression, anxiety, posttraumatic stress, and well-being), moral distress and moral reasoning, work-related changes and telehealth, organizational responses to COVID-19 concerns, and experiences with COVID-19 infections to self and to others. We recruited HCWs from across Canada through convenience snowball sampling to participate in either a short-form or long-form web-based survey at baseline. Respondents to the baseline survey are invited to complete a follow-up survey every 3 months, for a total of 18 months. 
Results: A total of 1926 participants completed baseline surveys between June 26 and December 31, 2020, and 1859 participants provided their emails to contact them to participate in follow-up surveys. As of July 2021, data collection is ongoing, with participants nearing the 6- or 9-month follow-up periods depending on their initial time of self-enrollment.

Conclusions: This protocol describes a study that will provide unique insights into the immediate and longitudinal impact of the COVID-19 pandemic on the dimensions of mental health, moral distress, health care delivery, and workplace environment of HCWs. The feasibility and acceptability of implementing a short-form and long-form survey on participant engagement and data retention will also be discussed.

International Registered Report Identifier (IRRID): DERR1-10.2196/32663

(JMIR Res Protoc 2021;10(9):e32663) doi: $10.2196 / 32663$

\section{KEYWORDS}

COVID-19; health care worker; pandemic; mental health; wellbeing; survey; design; longitudinal; prospective; protocol; challenge; impact; distress; perception

\section{Introduction}

\section{Background}

Throughout the COVID-19 pandemic, health care workers (HCWs) have served on the frontlines of disease management and response. In their roles, HCWs have experienced increased workloads, risks of redeployment, and exposure to SARS-CoV-2 while caring for the surge of patients. At the same time, HCWs may be concerned about the safety and well-being of themselves, their families, and their loved ones. Within a larger context, HCWs are situated in working environments that may be experiencing rapid changes, such as implementation of new safety protocols, adapting to telehealth service delivery, or contending with redeployment. These changes in the workplace are further compounded by increasingly challenging work environments, where HCWs may encounter difficult moral-ethical dilemmas (eg, tending to patients without adequate personal protective equipment (PPE), providing services on platforms unfamiliar to the provider and patients), which may have severe and enduring consequences for their mental health and well-being.

Research following the 2003 severe acute respiratory syndrome (SARS) epidemic illustrates the significant and persevering distress that HCWs may experience in the aftermath of an infectious outbreak. Among SARS survivors, HCWs experienced elevated symptoms of anxiety one month following SARS recovery [1] and higher levels of stress, depression, and anxiety at one-year postoutbreak [2] compared to non-HCW survivors. Evidence from the SARS epidemic also highlighted the vulnerability of those working on the frontlines, including job stress related to managing changes to working environments, feelings of loneliness and social isolation, and anxiety and fear in response to increased exposures to the virus [3,4]. In comparison to hospital administrative staff, frontline HCWs reported significantly higher psychological impairment, insomnia, and exhaustion [5].

Studies conducted early during the COVID-19 pandemic similarly found that HCWs on the front line were more severely distressed compared to nonfrontline HCWs [6-8]. In other cross-sectional studies, evidence also points to the devastating toll of the pandemic on the mental health of HCWs. In a study of nurses and physicians in Wuhan, China, over $60 \%$ of respondents reported concerning mental health symptoms across standardized measures of anxiety, depression, and sleep [9]. Similar increases in psychological distress, burnout, and worsened mental health were reported in frontline HCWs in other countries [10-13].

Some of the distress experienced by HCWs could be ethical or moral in nature. HCWs' experiences during the pandemic involve making difficult decisions that may not always be aligned with their ethical or moral values. These may involve tending to patients without appropriate PPE, balancing increases in patient caseloads and potentially compromising the quality of care provided, having to make difficult decisions to turn away patients without care due to shortages of hospital beds or ventilators, and disagreements or conflicts arising from the allocation of lifesaving treatments or vaccines [14,15]. Expanding beyond organizational levels, rapidly changing public health policies, and perceived delays in responses from leaders, employers, and municipal, provincial, and federal governments may perpetuate feelings of distrust and betrayal, further triggering complex emotional reactions. Indeed, moral distress may arise when individuals find themselves in difficult emotional states when the perceived ethical actions deviate from what they may be tasked to do. The frequency and impact of morally distressing events may be amplified during the current pandemic $[14,15]$. If unaddressed, these instances of moral distress can lead to moral injury, defined as the psychological distress resulting from transgressing one's moral beliefs or standards through action or inaction [16,17]. Despite evidence of mental distress, little empirical attention has been paid to the examination of moral-ethical dilemmas and associated moral distress during current and past epidemics and pandemics.

Further, the myriad of challenges HCWs face at the individual and organizational levels greatly threaten their physical and mental health, as well as their professional development. Specifically, increased rates of absenteeism reported by HCWs not only contribute to added burden of care for colleagues but more importantly highlight the systemic need for additional support for HCWs [18]. Meanwhile, reviews suggest that increased workloads, occupational stress and burnout, and organizational changes are expected to pose critical challenges in future to the long-term retention of HCWs [19]. Taken together, research is urgently needed to understand HCWs' experiences during the COVID-19 pandemic, including 
challenges and changes in the workplace, moral-ethical dilemmas, evolving occupational duties, standards of care, service delivery, and the effects of COVID-19 on dimensions of mental health and well-being.

\section{Research Aims}

This paper describes the protocol and initial response rates for a longitudinal study seeking to capture the unique experiences, challenges, and changes faced by HCWs during the COVID-19 pandemic. The study was launched in June 2020 and is ongoing. Future publications from this study will use the data collected to explore (1) the impact of COVID-19 on the mental well-being of HCWs, (2) perceptions of and satisfaction with delivery of care, and (3) how changes in work structure are tolerated among HCWs providing services.

\section{Methods}

\section{Summary of Design}

Our study employs an observational, prospective, longitudinal panel design using the web-based data collection platform Research Electronic Data Capture (REDCap). Participants completed questionnaires at baseline and will be completing follow-up questionnaires at 3-month intervals for a total of 18 months. Interested participants self-selected into an open survey and chose to complete a short version or long version of the survey at baseline. The protocols of this study were reviewed and approved by the research ethics board at Western University (WREM 115894) and Lawson Health Research Institute (REDA 9968). Details of the protocol are reported below following the general guidelines from the Checklist for Reporting of Results of Internet E-Surveys (CHERRIES) [20].

\section{Participant Selection and Recruitment}

A convenience snowball sampling approach is used to recruit HCWs. Recruitment methods include word of mouth, emails to professional networks, web-based advertisement through the Lawson Health Research Institute, social media, participant recruitment websites (eg, ParticipAid [21]), and targeted media releases. The representativeness of participants was monitored throughout the recruitment period. Recruitment efforts are adjusted to target specific regions or segments to improve the representation on dimensions of gender, region, and occupational distribution of health care workers within Canada. Participants include male and female English- and French-speaking HCWs with a minimum age of 18 years. HCWs are defined as individuals who provide health care treatment and advice based on formal training and experience or who work to directly support those providers in a clinical setting. Participating HCWs must be currently working in Canada or have worked in Canada as an HCW at some point in time between the start of the COVID-19 pandemic (March 2020) and the start of data collection (June 26, 2020). Participation in the study is voluntary, and participants are not compensated for survey completions.

\section{Procedure}

The study duration is catered to participant availability and varies depending on whether the participant selected the short-form or long-form survey (Table 1). Both English and French versions of the survey are available based on the language preference of participants. Validated scales in French are used where available, and in the absence of translated and validated versions, translation is completed by professional translators with certificates of translation provided. Informed consent is obtained through a Letter of Information (LOI), presented to participants at the beginning of the survey at baseline, and again during each of the follow-up surveys. The LOI is presented on REDCap, and participants are informed that their consent is implied should they proceed to the following pages of the survey.

A short-form version of the survey is available at baseline (approximately 10 minutes) and consists of 6 measures. A longer option, consisting of 12 measures, is available for participants who indicate they have the time (approximately 15-25 minutes). The long version can be completed immediately at baseline or returned within a 6-week window after beginning the baseline measures. Following baseline, participants are requested to complete a follow-up survey (approximately 15 minutes) every 3 months for a period of 18 months (see Table 1). To save time, participants can skip certain modules if there was no change from previous time points (eg, if their employment status did not change). Participants can also review and change answers before advancing to the following page. 
Table 1. Data collection tools for the short-form, long-form, and follow-up surveys.

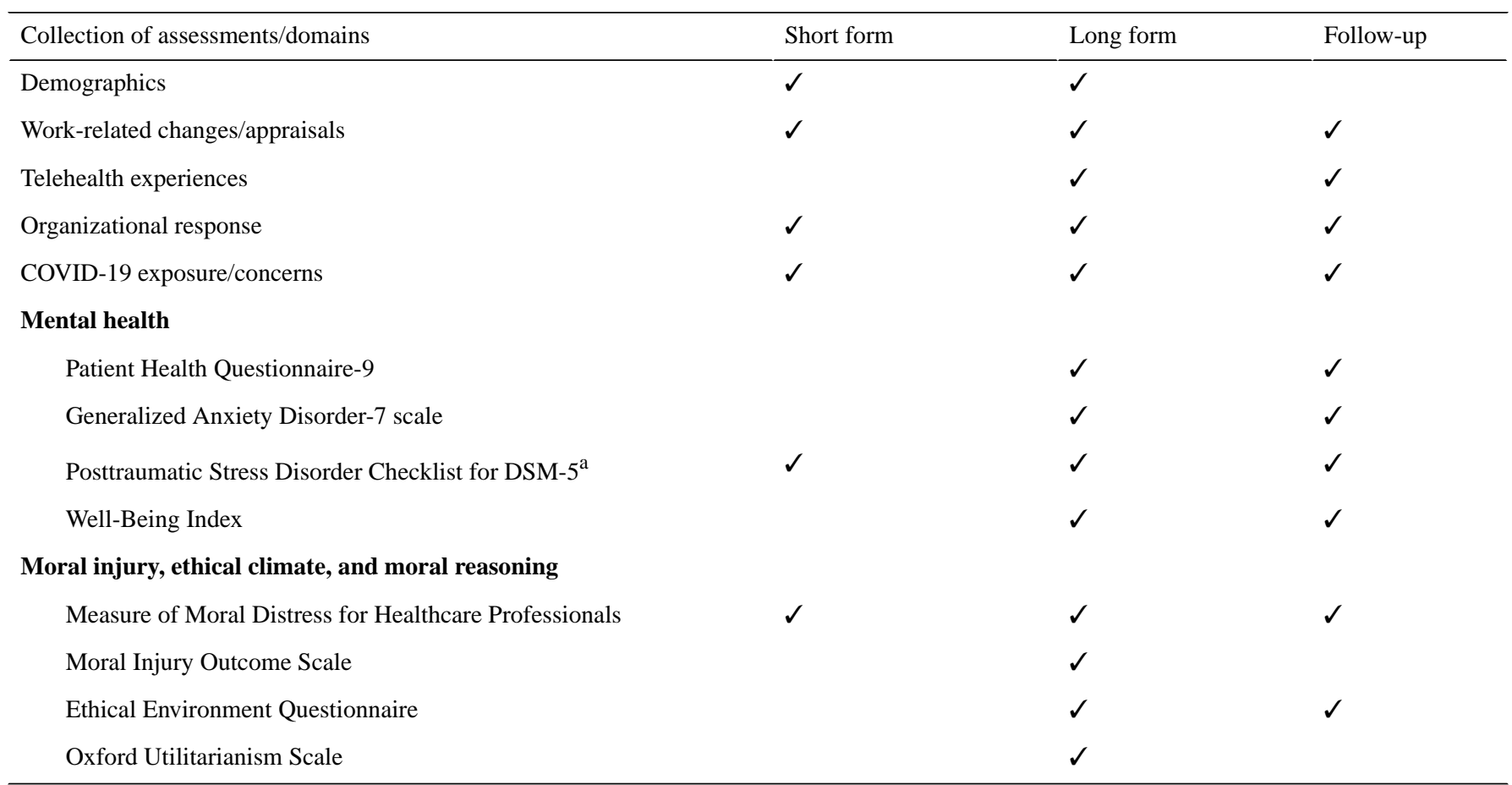

${ }^{a}$ DSM-5: Diagnostic and Statistical Manual of Mental Disorders, 5th edition.

\section{Measures}

The web-based survey was constructed in consultation with international research teams and collaborators to evaluate the crosscultural effects of the COVID-19 pandemic. Measures were selected to ensure comparability across countries. In addition, a literature search was conducted to identify potential novel measures related to the assessment of effects of the COVID-19 pandemic. The usability and functionality of the survey were tested by the research team, including adaptive questioning, branching functions, and longitudinal response collections. Questions were presented in the same order, as shown in Table 2. 
Table 2. Categories and types of questions included in the survey.

\begin{tabular}{ll}
\hline Category & Question types \\
\hline Basic demographics & $\begin{array}{l}\text { All participating } \mathrm{HCWs}^{\mathrm{a}} \text { are asked to report their province or territory of residence, age, and ethnicity. }{ }^{\mathrm{b}, \mathrm{c}} \text { Partici- } \\
\text { pants choosing to complete the long form questionnaire are also asked to report their gender, marital status, } \\
\text { characteristics of area of residence (eg, rural or city), and education level. }\end{array}$
\end{tabular}

Work-related/telehealth characteristics of area of residence (eg, rural or city), and education level.

Organizational response to COVID-19

COVID-19 exposure/concerns

Mental health questionnaires moral reasoning

Participants are asked about work demographics, the proportion of their time spent working on-site versus remotely, the proportion of their time working directly with patients, and whether they provided care to patients with suspected or confirmed COVID-19. HCWs providing services remotely are asked closed- and open-ended questions about the changes to their delivery of care and their experiences with telehealth. The telehealth questions were derived from a University of Missouri quality improvement survey and other research exploring the application of telehealth and telemedicine in various populations [22-25].

Participants are asked to self-report on the effectiveness and satisfaction with the support and communication of their organization in response to the COVID-19 pandemic. Items were drawn from the Pandemic Experiences and Perceptions Survey [26], a measure created in response to the COVID-19 pandemic. This survey measures organizational response to the pandemic in the domains of disruption, resource adequacy, COVID-19 risk perception, positive work life impact, and leadership [26]. For the purpose of our study, we are collecting data on the resource adequacy, risk perception, positive work life impact, and leadership domains.

Participants are asked to report their history of suspected or confirmed exposure to and infection with COVID19 individually and for family members, as well as any associated direct impact the infections had on them. The items were adapted from the Coronavirus Health Impact Survey, which was developed based on ongoing research and collaborations between the National Institute of Mental Health Intramural Research Program Mood Spectrum Collaboration, the Child Mind Institute, the New York State Nathan S Kline Institute for Psychiatric Research, and researchers from Johns Hopkins University [27].

Symptoms of mental distress are evaluated using self-report measures, including a measure of depression (the Patient Health Questionnaire-9 [28]), ${ }^{\mathrm{b}}$ posttraumatic stress disorder (the PTSD Checklist for DSM-5 [29]), generalized anxiety (the Generalized Anxiety Disorder-7 scale [30]), , and workplace well-being and burnout (WellBeing Index [31]). ${ }^{\mathrm{b}}$ Additional questions were included to determine the extent to which mental health symptoms may have been influenced or exacerbated by the pandemic.

Moral distress, moral injury, ethical workplace climate, and moral reasoning are assessed using self-report measures. Perceptions of the general ethical climate in the HCWs' workplaces will be evaluated using the Ethics Environment Questionnaire [32]. Individual experiences with specific morally distressing situations (eg, "Watch patient care suffer because of a lack of provider continuity") are evaluated using the Measure of Moral Distress for Healthcare Professionals [33]. Multidimensional moral injury is evaluated using the Moral Injury Outcome Scale ${ }^{\mathrm{b}}$ [34].

Moral-ethical decision-making tendencies are assessed using the Oxford Utilitarianism Scale ${ }^{\mathrm{b}}$ [35].

\footnotetext{
${ }^{\mathrm{a}} \mathrm{HCW}$ : health care worker.

${ }^{\mathrm{b}}$ Represents a measure that is available only on the long-form version of the web-based survey.

${ }^{\mathrm{c}}$ Ethnicity was added at a later time via an ethics amendment to the original protocol, and a portion of participants who are completing the 12-month follow-up survey will have the option to answer questions on their racial and ethnic background.
}

\section{Data Analytic Plan}

\section{Descriptive and Exploratory Analyses}

Mixed methods descriptive and exploratory analyses will be conducted to understand the state of mental well-being and moral distress of sampled HCWs. Quantitative descriptive statistics will examine age, gender, education, occupation, illness-related variables (eg, whether currently or formerly positive for COVID-19), psychological and moral variables, and satisfaction with telehealth. These will include general descriptive statistics, measures of internal consistency, correlational analyses, and group-based analyses on similarities and differences. Exploratory analyses will include hierarchical multivariate analyses, structural equation modelling, and cluster analyses to determine manifestations of mental and/or moral distress. Qualitative, open-ended data, including participant descriptions of changes to their care delivery, impact of moral distress, and general feedback on organizational support and

pandemic responses will be analyzed using content and thematic analysis.

\section{Longitudinal Analyses}

Data at baseline and each of the follow-up periods will be analyzed using exploratory and confirmatory mixed effects modeling and latent growth modeling. Longitudinal analyses will explore changes in dimensions of mental health, moral injury, and distress in relation to care delivery and/or work settings, organizational responses, and COVID-19-related changes and exposures over time.

\section{Results}

\section{Survey Completion and Representativeness}

A total of 1926 participants completed baseline surveys between June 26 and December 31, 2020. Of these, 1859 participants provided their emails for follow-up survey invitations. The majority $(71 \%, \mathrm{n}=1299)$ of baseline participants initially selected the long-form survey. Subsequent prompts encouraged 
participants completing the short-form surveys to complete the long version, which resulted in $25 \%$ conversion of those prompted and an overall increase in long-form survey data.
Figure 1 details the flow of survey completion from initial interest and engagement via survey links.

Figure 1. Participant flow for baseline completion rates. Info: information; LF: long-form survey; SF: short-form survey.

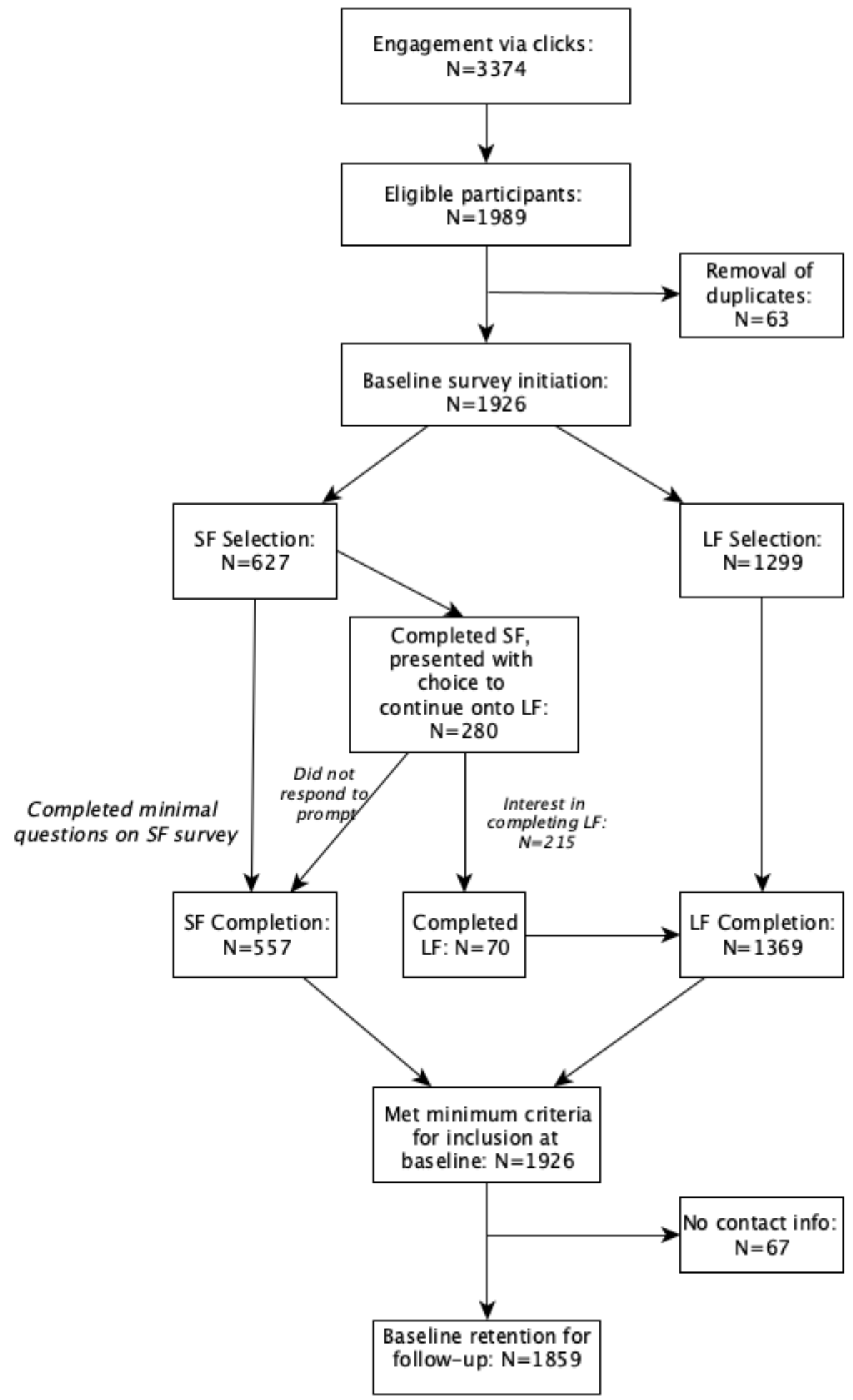

We further examined the preliminary representativeness of our baseline sample against a Canadian national database of health care workforce metadata with over 37 million health care workers on the distribution of top professions and genders [36]. Relative to the national sample, which comprises roughly $68 \%$ nurses and $14 \%$ physicians, our baseline sample from self-reported professions included $40.27 \%(\mathrm{n}=557)$ nurses and $3.90 \%(n=54)$ physicians, representing differences of $28 \%$ and $10 \%$, respectively. For other top health care disciplines, such as personal support workers, paramedics, physical therapists, and social workers, our sample was relatively representative, with differences in percentage distributions of $1.2 \%$ to $5.6 \%$. 
Finally, female nurses were underrepresented in our sample by a percentage difference of $32 \%$, whereas female $\mathrm{HCWs}$ in other disciplines and male $\mathrm{HCWs}$ were relatively representative in relation to the national sample (percentage differences of $<1 \%$ to $7 \%$ ).

\section{Participant Data Retention}

Depending on whether participants completed the short-form or long-form survey at baseline, completion time varied by approximately 12 minutes, with the long-form survey taking a median of 34 minutes and the short-form survey taking a median of 22 minutes to complete. Survey completion also varied by questionnaire. For the long-form survey, baseline completion for initial eligibility, work-related, and telehealth items were completed by nearly all participants (100\%), with participation declining to $<80 \%$ for COVID-19-related questions and to $<70 \%$ for mental health- and moral distress-related self-report questionnaires $(\mathrm{n} / \mathrm{N}$ values are not reported for the percentages

Figure 2. Completion rates of individual questionnaires at baseline.

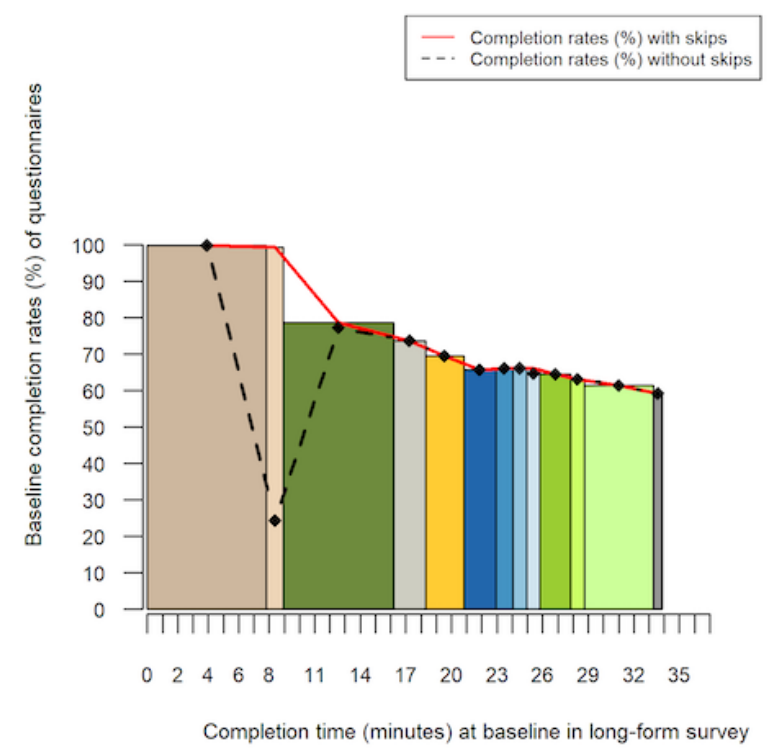

Finally, based on the date of self-selection and enrollment in the study, participants receive system-generated links to complete follow-up surveys every three months. The baseline retention with email addresses $(\mathrm{N}=1859)$ is being used as a reference point to evaluate subsequent participant retention, attrition, and sensitivity analyses during each of the follow-up periods. As of July 2021, a total of 848/1859 participants (45.6\%) had completed the 3-month follow-up, whereas 1011/1859 (54.4\%) missed the response window. Data collection is ongoing into the 6-month and 9-month follow-up periods. Full data collection for the longitudinal study is expected to be completed by August 8, 2022.

\section{Discussion}

This paper details the protocol for a longitudinal study that will examine the impact of the COVID-19 pandemic on the mental well-being of HCWs, with a focus on moral distress, perceptions here, as they represent changes in rates of completion as opposed to changes in sample size). This trend was similar for short-form questionnaire completion, but with a steeper decline, and with completion of the full set of questionnaires between $50 \%$ and $60 \%$ (see Figure 2). Although the dropout rate was comparable to those found in previous research evaluating completion rates of web-based surveys in relation to the length of the surveys, this study was able to maintain $100 \%$ initial completion for the first section of questions about work-related changes and responses to COVID-19, whereas others have reported $10 \%$ instantaneous dropout [37]. Furthermore, although previous studies examined participation under regular circumstances using university student samples, this study sampled HCWs who are likely time-restricted because of their busy schedules. Taken together, the choice between short- and long-form surveys and the option to convert to the long form is a promising approach to maximize participation and retention.

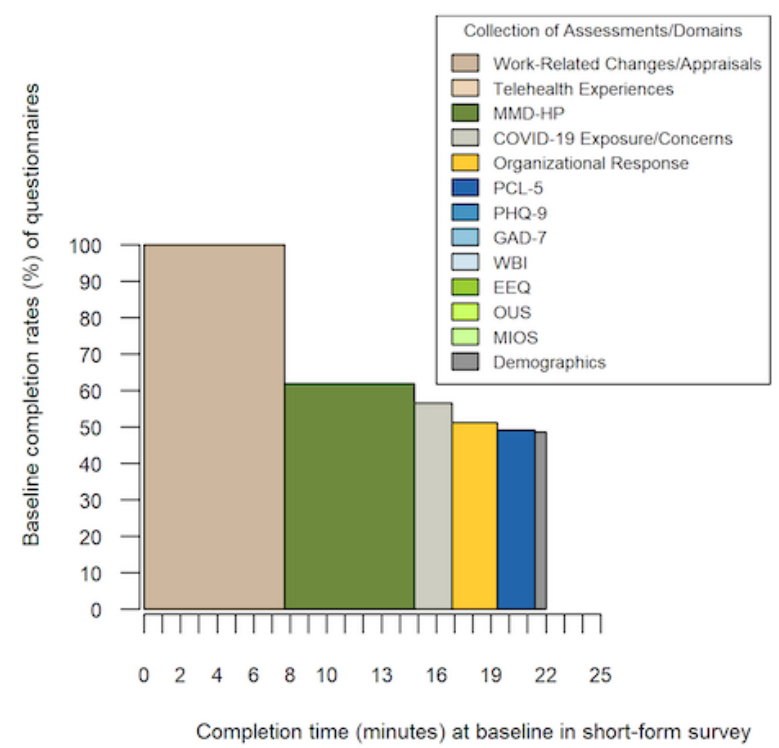

of and satisfaction with delivery of care, and perceptions of changes in work structure among HCWs providing services. Using convenience snowball sampling and diverse recruitment platforms, this study is reaching a large national sample of $\mathrm{HCW}$ from a range of disciplines and backgrounds. Participants recruited are relatively diverse and comparable to national samples of HCW distributions. Following baseline completion, we retained $1859 \mathrm{HCW}$ s for subsequent longitudinal follow-ups. The longitudinal data will provide important profiles of HCWs during key milestones of the pandemic in Canada and offer insights in understanding and predicting the development or worsening of mental health and moral injury over time as the pandemic persists. In particular, the collected data will shed light on the organizational and environmental stressors and their associations with changes in the experiences of HCWs with moral dilemmas, moral distress, and mental well-being. 
A strength of this study that may contribute to the large sample size for both recruitment and retention is the option to complete either the short or long form of the survey. With the choice to select either form, we sought to reduce barriers of participation as a result of survey fatigue and self-perceived time restrictions to accommodate the busy schedules of HCWs. To further encourage completion of the long-form survey, we also implemented prompts to encourage those who completed the short-form survey to complete the long-form survey in subsequent follow-ups. This option yielded a $25 \%$ success rate in conversion to the long-form survey. This represents a relatively novel approach, which was designed to maximize survey retention while minimizing barriers [38]. A challenge in this process was the time difference in completion between short- and long-form surveys. Based on data completion rates and times at baseline, the difference between the two surveys is estimated to range between 5 and 15 minutes, with the short-form survey taking a median of 22 minutes to complete. Given the initial time commitment required for the short-form survey, this may have discouraged some of the participants from converting to the long-form survey based on their perceived time restraints.

As of July 2021, data collection is ongoing, with participants nearing the 6- or 9-month follow-up periods depending on their initial time of self-enrollment. This study demonstrates the utility and feasibility of offering both a short-form and long-form survey for the collection of prospective, longitudinal data from HCWs. This format of recruitment and data collection may be useful when implemented with other populations experiencing time restrictions or busy schedules. Finally, this study will offer key insights into the mental well-being and moral challenges of HCWs as they cope with the ongoing pandemic. The findings will lend a voice to the HCWs and their unique experiences of challenges and change during the protracted pandemic and associated restrictions. Knowledge gained will better equip us to anticipate and prepare for future challenges, such as long-term support and retention of HCWs. Armed with this knowledge, policy makers and clinicians can make evidence-based decisions to prevent and mitigate the risks to the psychological well-being of HCWs both generally and specifically as they recover from the pandemic.

\section{Acknowledgments}

This research received no direct funding or support from any funding agency in the public, commercial, or not-for-profit sector. This research initiative is conducted in partnership with the Centre of Excellence on Post-Traumatic Stress Disorder and Related Mental Health Conditions ( $\mathrm{CoE})$; as collaborators and partners, CoE is covering partial expenses related to the survey creation and administration.

\section{Conflicts of Interest}

None declared.

\section{References}

1. Cheng S, Wong CW, Tsang J, Wong KC. Psychological distress and negative appraisals in survivors of severe acute respiratory syndrome (SARS). Psychol Med 2004 Oct;34(7):1187-1195 [FREE Full text] [doi: 10.1017/s0033291704002272] [Medline: 15697045$]$

2. Lee AM, Wong JG, McAlonan GM, Cheung V, Cheung C, Sham PC, et al. Stress and psychological distress among SARS survivors 1 year after the outbreak. Can J Psychiatry 2007 Apr 01;52(4):233-240. [doi: 10.1177/070674370705200405] [Medline: 17500304]

3. Maunder R, Hunter J, Vincent L, Bennett J, Peladeau N, Leszcz M, et al. The immediate psychological and occupational impact of the 2003 SARS outbreak in a teaching hospital. CMAJ 2003 May 13;168(10):1245-1251 [FREE Full text] [Medline: 12743065$]$

4. Maunder R, Lancee WJ, Rourke S, Hunter JJ, Goldbloom D, Balderson K, et al. Factors associated with the psychological impact of severe acute respiratory syndrome on nurses and other hospital workers in Toronto. Psychosom Med 2004;66(6):938-942 [FREE Full text] [doi: 10.1097/01.psy.0000145673.84698.18] [Medline: 15564361 ]

5. Bai Y, Lin CC, Lin CY, Chen JY, Chue CM, Chou P. Survey of stress reactions among health care workers involved with the SARS outbreak. Psychiatr Serv 2004 Sep;55(9):1055-1057. [doi: 10.1176/appi.ps.55.9.1055] [Medline: 15345768]

6. Dai Y, Hu G, Xiong H, Qiu H, Yuan X. Psychological impact of the coronavirus disease 2019 (COVID-19) outbreak on healthcare workers in China. medRxiv Preprint posted online on March 6, 2020. [doi: 10.1101/2020.03.03.20030874]

7. Lai J, Ma S, Wang Y, Cai Z, Hu J, Wei N, et al. Factors associated with mental health outcomes among health care workers exposed to coronavirus disease 2019. JAMA Netw Open 2020 Mar 02;3(3):e203976 [FREE Full text] [doi: 10.1001/jamanetworkopen.2020.3976] [Medline: 32202646]

8. De Kock JH, Latham HA, Leslie SJ, Grindle M, Munoz S, Ellis L, et al. A rapid review of the impact of COVID-19 on the mental health of healthcare workers: implications for supporting psychological well-being. BMC Public Health 2021 Jan 09;21(1):104 [FREE Full text] [doi: 10.1186/s12889-020-10070-3] [Medline: 33422039]

9. Huang Y, Zhao N. Generalized anxiety disorder, depressive symptoms and sleep quality during COVID-19 outbreak in China: a web-based cross-sectional survey. Psychiatry Res 2020 Jun;288:112954 [FREE Full text] [doi: 10.1016/j.psychres.2020.112954] [Medline: 32325383] 
10. Kachadourian LK, Feder A, Murrough JW, Feingold JH, Kaye-Kauderer H, Charney D, et al. Transdiagnostic psychiatric symptoms, burnout, and functioning in frontline health care workers responding to the COVID-19 pandemic. J Clin Psychiatry 2021 Apr 27;82(3). [doi: 10.4088/jcp.20m13766]

11. Orrù G, Marzetti F, Conversano C, Vagheggini G, Miccoli M, Ciacchini R, et al. Secondary traumatic stress and burnout in healthcare workers during COVID-19 outbreak. Int J Environ Res Public Health 2021 Jan 05;18(1):337 [FREE Full text] [doi: 10.3390/ijerph18010337] [Medline: 33466346]

12. Shreffler J, Petrey J, Huecker M. The impact of COVID-19 on healthcare worker wellness: a scoping review. West J Emerg Med 2020 Aug 17;21(5):1059-1066 [FREE Full text] [doi: 10.5811/westjem.2020.7.48684] [Medline: 32970555]

13. Spoorthy M, Pratapa SK, Mahant S. Mental health problems faced by healthcare workers due to the COVID-19 pandemic-A review. Asian J Psychiatr 2020 Jun;51:102119 [FREE Full text] [doi: 10.1016/j.ajp.2020.102119] [Medline: 32339895]

14. COVID-19 and moral distress: setting the stage. Canadian Medical Association. URL: https://www.cma.ca/sites/default/ files/pdf/Moral-Distress-E.pdf [accessed 2021-07-20]

15. Roycroft M, Wilkes D, Pattani S, Fleming S, Olsson-Brown A. Limiting moral injury in healthcare professionals during the COVID-19 pandemic. Occup Med (Lond) 2020 Jul 17;70(5):312-314 [FREE Full text] [doi: 10.1093/occmed/kqaa087] [Medline: $\underline{\text { 32428213] }}$

16. Shay J. Odysseus in America: Combat Trauma and the Trials of Homecoming. New York, NY: Simon \& Schuster; Nov 25, 2003.

17. Litz BT, Stein N, Delaney E, Lebowitz L, Nash WP, Silva C, et al. Moral injury and moral repair in war veterans: a preliminary model and intervention strategy. Clin Psychol Rev 2009 Dec;29(8):695-706. [doi: 10.1016/j.cpr.2009.07.003] [Medline: $\underline{19683376}$ ]

18. Tujjar O, Simonelli M. Absenteeism of frontline healthcare workers during Covid-19: the need for a framework of support. SN Compr Clin Med 2020 Oct 28;19:1-3 [FREE Full text] [doi: 10.1007/s42399-020-00609-1] [Medline: 33134848]

19. Jamebozorgi AH, Agoush L, NooriHekmat S, Shokri A, Sadatmoosavi A, Shirvani M, et al. Coronavirus and its impacts on health workers retention: a systematic review and meta-synthesis. Research Square Preprint posted online on February 23, 2021. [FREE Full text] [doi: 10.21203/rs.3.rs-220298/v1]

20. Eysenbach G. Improving the quality of Web surveys: the Checklist for Reporting Results of Internet E-Surveys (CHERRIES). J Med Internet Res 2004 Sep 29;6(3):e34 [FREE Full text] [doi: 10.2196/jmir.6.3.e34] [Medline: 15471760]

21. ParticipAid. URL: https://participaid.co/ [accessed 2021-09-09]

22. Becevic M, Boren S, Mutrux R, Shah Z, Banerjee S. User satisfaction with telehealth: study of patients, providers, and coordinators. Health Care Manag 2015;34(4):337-349 [FREE Full text] [doi: 10.1097/hcm.0000000000000081]

23. Brown S. Primary care provider's perceptions of telehealth adoption (Order No AAI10163004). APA PsycInfo. 2017.

24. Hicks L, Fleming DA, Desaulnier A. The application of remote monitoring to improve health outcomes to a rural area. Telemed J E Health 2009 Sep;15(7):664-671 [FREE Full text] [doi: 10.1089/tmj.2009.0009] [Medline: 19694598]

25. Sandberg J, Trief PM, Izquierdo R, Goland R, Morin PC, Palmas W, et al. A qualitative study of the experiences and satisfaction of direct telemedicine providers in diabetes case management. Telemed J E Health 2009 Oct;15(8):742-750 [FREE Full text] [doi: 10.1089/tmj.2009.0027] [Medline: 19780691]

26. Leiter MP. Mind Garden. URL: https://www.mindgarden.com/346-pandemic-experiences-perceptions-survey [accessed 2021-06-16]

27. Merikangas K, Milham M. The CoRonavIruS Health Impact Survey (CRISIS) V0.1. GitHub. URL: https://github.com/ nimh-mbdu/CRISIS/tree/d94bae3eba7b225f89fb310eae881d1d73ee9126 [accessed 2021-09-09]

28. Spitzer RL, Kroenke K, Linzer M, Hahn SR, Williams JBW, deGruy FV, et al. Health-related quality of life in primary care patients with mental disorders: results from the PRIME-MD 1000 study. JAMA 1995 Nov 15;274(19):1511. [doi: 10.1001/jama.1995.03530190025030]

29. Weathers FW, Litz BY, Keane TM, Palmieri PA, Marx BP, Schnurr PP. PTSD Checklist for DSM-5 (PCL-5). US Department of Veterans Affairs. 2013. URL: https://www.ptsd.va.gov/professional/assessment/adult-sr/ptsd-checklist.asp [accessed 2021-09-09]

30. Spitzer RL, Kroenke K, Williams JBW, Löwe B. A brief measure for assessing generalized anxiety disorder: the GAD-7. Arch Intern Med 2006 May 22;166(10):1092-1097. [doi: 10.1001/archinte.166.10.1092] [Medline: 16717171]

31. Dyrbye L, Satele D, Shanafelt T. Ability of a 9-item well-being index to identify distress and stratify quality of life in US workers. J Occup Environ Med 2016;58(8):810. [doi: 10.1097/jom.0000000000000798]

32. McDaniel C. Development and psychometric properties of the Ethics Environment Questionnaire. Med Care 1997 Sep;35(9):901-914. [doi: 10.1097/00005650-199709000-00003] [Medline: 9298079]

33. Epstein EG, Whitehead PB, Prompahakul C, Thacker LR, Hamric AB. Enhancing understanding of moral distress: the measure of moral distress for health care professionals. AJOB Empir Bioeth 2019 Apr 19;10(2):113-124. [doi: 10.1080/23294515.2019.1586008] [Medline: 31002584]

34. Yeterian J, Berke DS, Carney JR, McIntyre-Smith A, St Cyr K, King L, Members of the Moral Injury Outcomes Project Consortium. Defining and measuring moral injury: rationale, design, and preliminary findings from the Moral Injury Outcome Scale Consortium. J Trauma Stress 2019 Jun;32(3):363-372 [FREE Full text] [doi: 10.1002/jts.22380] [Medline: 30947372] 
35. Kahane G, Everett JAC, Earp BD, Caviola L, Faber NS, Crockett MJ, et al. Beyond sacrificial harm: a two-dimensional model of utilitarian psychology. Psychol Rev 2018 Mar;125(2):131-164 [FREE Full text] [doi: 10.1037/rev0000093] [Medline: 29265854]

36. Health Workforce Database metadata. Canadian Institute for Health Information. URL: https://www.cihi.ca/en/ health-workforce-database-metadata [accessed 2021-06-16]

37. Hoerger M. Participant dropout as a function of survey length in internet-mediated university studies: implications for study design and voluntary participation in psychological research. Cyberpsychol Behav Soc Netw 2010 Dec;13(6):697-700 [FREE Full text] [doi: 10.1089/cyber.2009.0445] [Medline: 21142995]

38. Teague S, Youssef GJ, Macdonald JA, Sciberras E, Shatte A, Fuller-Tyszkiewicz M, SEED Lifecourse Sciences Theme. Retention strategies in longitudinal cohort studies: a systematic review and meta-analysis. BMC Med Res Methodol 2018 Nov 26;18(1):151 [FREE Full text] [doi: 10.1186/s12874-018-0586-7] [Medline: 30477443]

\author{
Abbreviations \\ CoE: Centre of Excellence on Post-Traumatic Stress Disorder and Related Mental Health Conditions \\ HCW: health care worker \\ LOI: Letter of Information \\ PPE: personal protective equipment \\ REDCap: Research Electronic Data Capture \\ SARS: severe acute respiratory syndrome
}

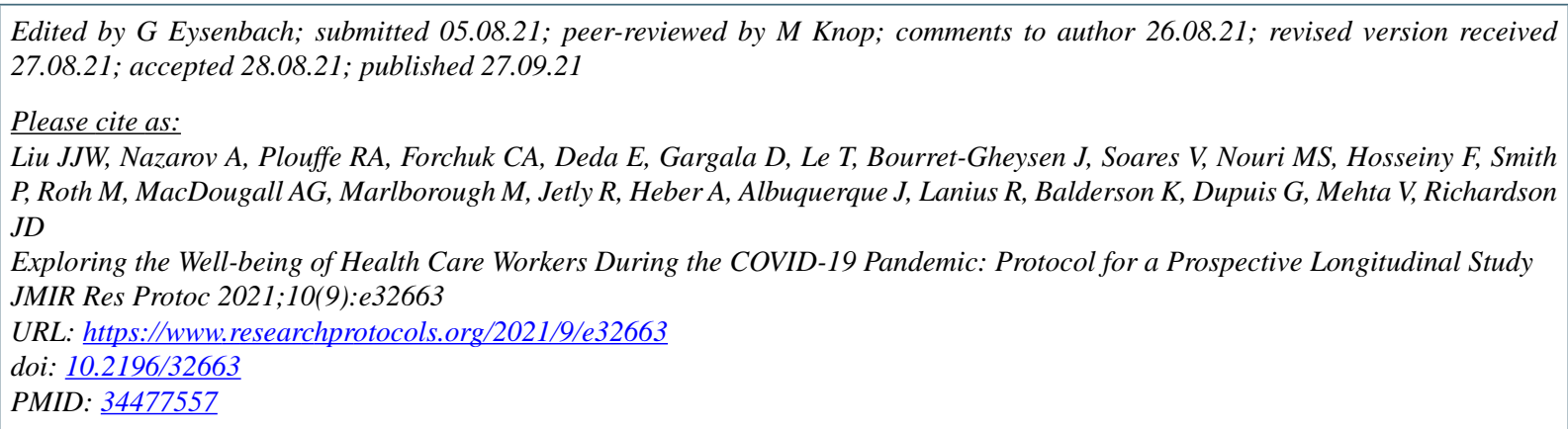

CJenny J W Liu, Anthony Nazarov, Rachel A Plouffe, Callista A Forchuk, Erisa Deda, Dominic Gargala, Tri Le, Jesse Bourret-Gheysen, Vanessa Soares, Maede S Nouri, Fardous Hosseiny, Patrick Smith, Maya Roth, Arlene G MacDougall, Michelle Marlborough, Rakesh Jetly, Alexandra Heber, Joy Albuquerque, Ruth Lanius, Ken Balderson, Gabrielle Dupuis, Viraj Mehta, J Don Richardson. Originally published in JMIR Research Protocols (https://www.researchprotocols.org), 27.09.2021. This is an open-access article distributed under the terms of the Creative Commons Attribution License (https://creativecommons.org/licenses/by/4.0/), which permits unrestricted use, distribution, and reproduction in any medium, provided the original work, first published in JMIR Research Protocols, is properly cited. The complete bibliographic information, a link to the original publication on https://www.researchprotocols.org, as well as this copyright and license information must be included. 